\title{
Design optimization of a bioreactor for ethanol production using CFD simulation and genetic algorithms
}

\author{
E. R. C. Góis \& P. Seleghim Jr. \\ Thermal and Fluids Engineering Laboratory, \\ University of São Paulo, Brazil
}

\begin{abstract}
The increase of the use of biofuels, like bioethanol, has presented new challenges to engineering problems. The optimization of bioreactors is crucial to improve the bioethanol extraction and to avoid stagnant zones in the flow that can compromise the chemical reactions involved in the process. This paper presents a solution using Computational Fluid Dynamics tools coupled with Genetics Algorithm to find an improved reactor structure. The preliminary results show that the influence of the height outlet tube alone is able to modify completely the flow pattern inside the reactor, improving the efficiency of the reactor.

Keywords: bioethanol, bioreactor, CFD, genetic algorithms, optimization.
\end{abstract}

\section{Introduction}

The search for new ways to provide fuel for the society has been constantly increasing. One of the great challenges for scientists and academic researchers is to provide fuels without jeopardizing the environment. An interesting alternative is the bioethanol production from sugar cane or other kinds of biomass, like corn, beet, etc.

The production of sugar cane ethanol is economically feasible only in large scale, such as 45 thousand liters per hour, for instance. At these scales, any efficiency improvement can result in a very significant overall optimization in terms of production rates and environmental impacts as well. Due to the difficulty to do experimental analyses, the expensive materials that are used and the time limitations, Computational Fluid Dynamics (CFD) tools are a useful alternative to study the flow inside reactors and bioreactors, and to better define 
their design principles. CFD tools have been applied in different kinds of reactor and bioreactor simulations. For example, Patwardhan et al. [1] studied the relationship between geometry and operating conditions of an industrial gasliquid stirred reactor and had a good prediction of the residence time distribution of the industrial scale reactor. CFD tools were applied to perform stirred solidliquid reactors [3]. The study of suspension of solid particles and the influence of them in the flow were considered by Shao et al. [4].

Most of the studies, however, were developed for small-scale reactors. Largescale bioreactors, usually applied at bioethanol production, must be studied more carefully because the rule of fluid velocity profile, shear stress, and other physical conditions may be considerably different compared with small-scale bioreactors. There are two possible ways of extracting ethanol from sugar cane: the first generation ethanol and the second generation ethanol.

First generation ethanol is produced directly from the sugar cane juice. The sugar cane juice is submitted to a fermentation process, which results in sugar or ethanol. In this case, a bioreactor is used because there is the necessity to use live organisms as a means of process acceleration. For the second generation ethanol, the principle is to reuse the bagasse resulting from the sugar cane juice. In this case, it is possible to use of a thermo-pressurized reactor with a mechanic process.

In both cases, the reactor should be able to provide optimum conditions to the chemical or mechanical process. The design of the reactor has a great influence in the flow conditions, with respect to mass transfer, shear stress, mixing, control of $\mathrm{pH}$, temperature and substrate conditions [2].

Some flow conditions can be a bad influence on the chemical and mechanical reactions inside the bioreactor. Stagnant zones keep the sugar cane juice inside the reactor for longer or shorter than necessary. In both cases, stagnant zones are prejudicial to bioethanol production, consequently, the velocity flow inside the bioreactor must be controlled.

An alternative to control the flow inside the reactor and get yields improvements is to modify structural parameters. The geometry definition has a great influence in the flow and consequently in the chemical reaction. Several methods have been used in order to determine the best way to optimize and obtain the best structural parameters. Two classes of search methods have dominated the estimation for structural parameters: the deterministic and stochastic methods [5].

The deterministic methods have some limitations when applied in some recent engineering problems. This kind of methods, despite being simpler to implement than stochastic methods, requires the calculation of second order derivatives and are strongly dependents on the initial approximation for the solution. In some cases there is more than one objective function, in other words, more than one parameter to be optimized. Sometimes these parameters are conflicting. For example, Mokeddem and Khellaf [6] tried to find an optimal feeding profile in feed-batch reactors, intending to maximise the amount of a protein used while simultaneously minimizing the volume of inducer employed, minimizing the material costs. In these cases, the most convenient way to 
evaluate the best structural parameters is applying a stochastic method, like genetic algorithms.

Genetic algorithms are a class of stochastic methods solving complex optimization problems of the real world [7]. These methods are based on mechanisms of natural selection and population genetics [8].

The aim of this paper is to provide an optimized structure design for a continuous reactor applied to ethanol production from sugar cane juice. For this, different heights outlet tube were tested, intending minimize the media of the velocities inside the bioreactor. To find the ideal structural parameters, the flow simulations are coupled with an optimization process based on genetic algorithms.

\section{Methodology}

The ANSYS CFX 13.0 commercial software was used to simulate the flow inside the bioreactor. To perform the flow inside the bioreactor, the selected fluid was initially water. The modelling equations were the Navier-Stokes equations, given by eqn. (1) and eqn. (2):

$$
\begin{gathered}
\frac{\partial \rho}{\partial t}+\nabla \cdot(\rho U)=0 \\
\frac{\partial(\rho U)}{\partial t}+\nabla \cdot(\rho U X U)=-\nabla p+\nabla \tau+S N
\end{gathered}
$$

where $\tau$ is given by:

$$
\tau=\mu\left(\nabla U+(\nabla U)^{T}-\frac{2}{3} \delta \nabla \cdot U\right)
$$

where $U$ is the normal velocity, $p$ the pressure, $t$ the time, $\rho$ de density and SN the forcing terms.

Due the computational efforts required, some simplifications in the flow and the mesh were assumed. This simplification does not affect the objective of this study. A steady flow simulation was performed. The flow was isothermal and incompressible. Turbulence effects were taken in account with the K- $\varepsilon$ model, and the Navier-Stokes equations were solved using the Finite Volume method. The advection scheme was solved using High Resolution Method.

To perform the first simulation, an initial structure was used. Figure 1 shows the mentioned structure, composed by one tube for fluid entrance, one tube for fluid exit, and a cylindrical reservoir. The tube diameter for flow entrance and exit are both $5 \mathrm{~cm}$, and the reservoir volume is 887 liters. These dimensions were considered according to the requirements for ethanol sugar cane production. The reservoir height was $1.5 \mathrm{~m}$ and the initial outlet tube was $1 \mathrm{~m}$.

A tetrahedral mesh with about 2787 nodes and 13963 cells was used in this simulation, as shown in Fig. 2.The inlet velocity applied was $0.1 \mathrm{~m} / \mathrm{s}^{2}$ and a steady analysis was performed, and the outlet pressure was defined as $0 \mathrm{~Pa}$. 


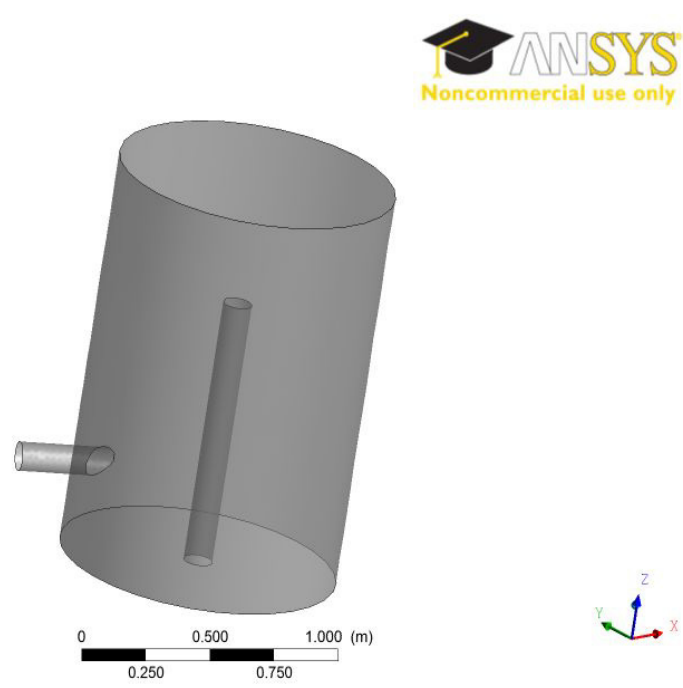

Figure 1: $\quad$ Structure used for initial tests.

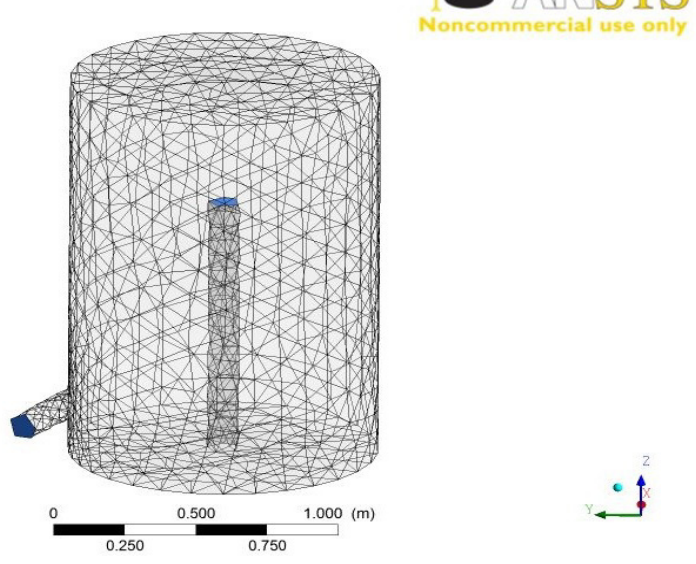

Figure 2: Domain discretization.

Besides the fluid analysis, the aim of this study was to optimize the bioreactor structure, improving the bioreactor efficiency by velocity controlling. As discussed previously, genetic algorithms are a useful tool in the optimizing process, allowing multi-objective optimization.

The results presented in this paper were performed applying the MultiObjective Genetic Algorithm MOGA-II [9].MOGA II is an improved version of MOGA (Fonseca and Fleming [10]) and uses five different operators for 
reproduction: selection, classical cross-over, directional cross-over, mutation and elitism. At each step of the reproduction process, one of the five operators is chosen and applied to the current individual. The directional cross-over assumes that a direction of improvement can be detected comparing the fitness values of two individual references, and this comparison is made in the same generation. This kind of cross-over helps the algorithm convergence. The MOGA II is implemented in the Mode FRONTIER 4.3 commercial software. This software allows the coupling between the Ansys CFX 13 solver and MOGA II, automating the process. Schematically, the coupling between CFX and Mode FRONTIER works in this order: structure generation, mesh generation, fluid solver, optimization and new parameters definition, new structure generation, and so successively, until the stop criterion is reached.

\section{Results}

MOGA II was employed to solve the optimization problems with the parameters shown in Table 1.

Forty-six height outlet possibilities were performed. All the height outlet tube possibilities and feasibilities are shown in Fig. 3.

Table 1: $\quad$ MOGA II parameters.

\begin{tabular}{|c|c|}
\hline & Parameters \\
\hline Number of generations & 100 \\
\hline Probability of directional Cross-Over & 0.5 \\
\hline Probability of selection & 0.05 \\
\hline Probability of mutation & 0.1 \\
\hline
\end{tabular}

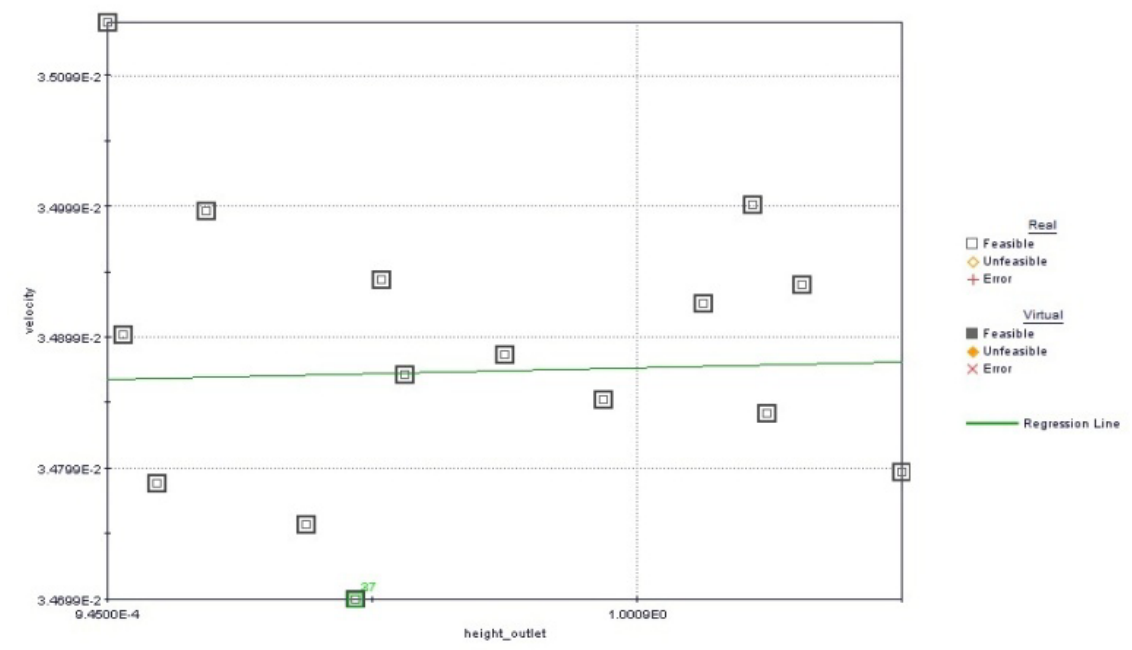

Figure 3: Height outlet tube and respective velocities. 
After the evaluation of 46 different designs, the results applying MOGA II show that the ideal height outlet tube for minimize the media of velocities is 0.46 $\mathrm{cm}$.

The streamlines for the optimized structure is shown in Fig. 4. The height outlet tube contributes for an organized flow inside the bioreactor.

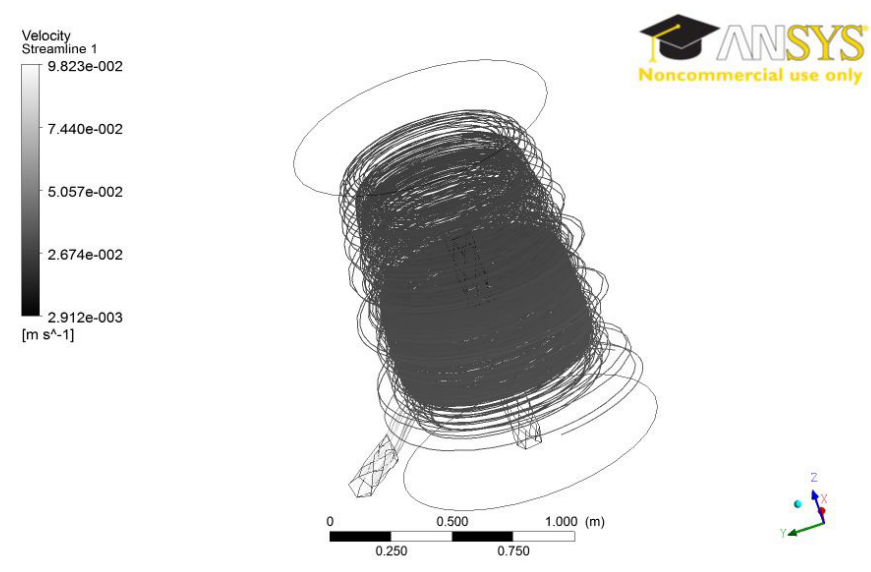

Figure 4: Streamlines for optimized case.

\section{Conclusions}

Propose new ways to obtain bioethanol from sugar cane and improve the existing ones are a great challenge for engineering applications. Considering a bioreactor applied in bioethanol production, this paper presents the results of an investigation about the influence of the structural parameters in the flow and, consequentially, in the chemical reactions in the bioethanol production. Were tested 46 different values of outlet tube height, and the best value was determined. To select the best parameters a Genetic Algorithm, MOGA II, was applied by Mode FRONTIER 4.3 commercial software coupled with Ansys CFX 13. Despite the initial tests have computational efforts limited, due the intention to execute the coupling between the CFD analysis and the optimization process, a good performance was obtained.

The knowledge about the optimized structure provides a bioreactor yield improvement, material economy and environmental impact reduction. Future works are concerned in tests validation and verification through an experimental prototype fabrication and improvements on CFD modeling.

\section{References}

[1] Patwardhan A. W., Joshi J. B., Fotedar S. \& Mathew T., Optimization of gas-liquid reactor using computational fluid dynamics. Chemical Engineering Science.60 , pp. 3081-3089 (2005) 
[2] Xia J., Wang S., Zhang S. \& Zhong J., Computational investigation of fluid dynamics in a recently developed centrifugal impeller bioreactor. Biochemical Engineering Journal.38 , pp. 406-413 (2008)

[3] Micale G., Rizutti L. \& Brucato A., CFD simulation of particle suspension height in stirred vessels. Chemical Engineering Research and Design.82, pp. 1204-1213 (2004)

[4] Shao X., Lynd L., Bakker A., LaRoche R. \& Wyman C., Reactor scale-up for biological conversion of cellusosic biomass to ethanol. Bioprocess Biosystem Engineering. 33, pp. 485-493. (2010)

[5] Rezende M. C. A. F., Costa C. B. B., Costa A. C., Maciel M. R. W. \&Maciel R. F., Optimization of a large scale industrial reactor by genetic algorithms. Chemical Engineering Science.63 , pp. 330-341 (2008)

[6] Mokeddem D. \&Khellaf A., Optimal feeding profile in fed-batch bioreactors using a genetic algorithm. International Journal of Production Research. 48, pp. 6125-6135. (2010)

[7] Kordabadi H. \& Jahanmiri A., Optimization of methanol synthesis reactor using genetic algorithm. Chemical Engineering Journal.108 , pp. 249-255 (2005)

[8] Dasgupta D., \& Michalewicz, Z., (eds). Evolutionary algorithms in engineering applications, Springer: New York, 1997.

[9] Poloni A., Giurgevich A., Onesti L. \& Pediroda V., Hybridization of a multi-objective genetic algorithm, a neural network and a classical optimizer for a complex design in fluid dynamics. Computational methods in applied mechanics and engineering. 186, pp. 403-420. (2000)

[10] Fonseca, C.M. \& Fleming P.J., Multi objective Genetic Algorithms. Proc. of the IEE Coloquium on Genetic Algorithm for Control Systems Engineering, IEEE: London UK, pp. 1-6, 1993. 